\title{
Supporting Community Health Workers in India through Voice- and Web-Based Feedback
}

\author{
Brian DeRenzi ${ }^{\dagger}$ Nicola Dell ${ }^{\ddagger} \quad$ Jeremy Wacksman $^{\phi} \quad$ Scott Lee $^{\Psi} \quad$ Neal Lesh $^{\phi}$ \\ $\dagger$ University of Cape Town \\ $\stackrel{\ddagger \text { Cornell Tech }}{\text { nixdell@ cornell.edu }}$ \\ bderenzi@cs.uct.ac.za \\ $\phi$ Dimagi, Inc. \\ jwacksman,nlesh \\ @ dimagi.com

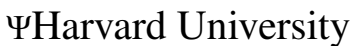
slee@hbs.edu

\begin{abstract}
Our research aims to support community health workers (CHWs) in low-resource settings by providing them with personalized information regarding their work. This information is delivered through a combination of voice- and webbased feedback that is derived from data already collected by CHWs. We describe the in situ participatory design approach used to create usable and appropriate feedback for low-literate CHWs and present usage data from a 12-month study with 71 CHWs in India. We show how the system supported and motivated CHWs, and how they used both the web- and voice-based systems, and each of the visualizations, for different reasons. We also show that the comparative feedback provided by the system introduced elements of competition that discouraged some CHWs while motivating others. Taken together, our findings suggest that providing personalized voice- and web-based feedback could be an effective way to support and motivate CHWs in low-resource settings.
\end{abstract}

\section{Author Keywords}

ICTD; HCI4D; CHW; ASHA; community health; mHealth.

\section{INTRODUCTION}

The critical lack of trained medical professionals in many low-income countries has resulted in the establishment of community health programs that aim to provide vulnerable populations with access to essential health services. The success of community health programs hinges on the work of lightly-trained community health workers (CHWs), who are members of local communities that receive basic medical training and who then assess, treat, and refer patients according to established health protocols. For many community members, CHWs are the primary link to the broader public health infrastructure. However, CHWs operating in low-resource contexts face a diverse set of challenges as they work to deliver health care to their communities, including poor infrastructure, low levels of education and literacy, limited training and supervision, isolation, lack of support, and

Permission to make digital or hard copies of all or part of this work for personal or classroom use is granted without fee provided that copies are not made or distributed for profit or commercial advantage and that copies bear this notice and the full citation on the first page. Copyrights for components of this work owned by others than ACM must be honored. Abstracting with credit is permitted. To copy otherwise, or republish, to post on servers or to redistribute to lists, requires prior specific permission and/or a fee. Request permissions from permissions@acm.org.

CHI 2017, May 06-11, 2017, Denver, CO, USA

(C) 2017 ACM. ISBN 978-1-4503-4655-9/17/05 .. \$15.00

DOI: http: //dx .doi .org/10.1145/3025453. 3025514 the need to balance $\mathrm{CHW}$ work with other duties such as agriculture, child care, and family responsibilities.

In an effort to ameliorate some of these challenges, researchers in the fields of HCI for Development (HCI4D) and Information and Communication Technologies for Development (ICTD) have been exploring ways in which mobile devices could support and strengthen CHW programs. Examples include providing CHWs with decision support [11], job aids [15], health information [21, 27], and reminder messages $[10,22]$. However, the majority of these systems deliver generic messages or videos that are not personalized to the CHW's individual context. A range of data collection systems also allow CHWs to collect and submit data to a central database $[4,5,16,18]$. However, although the collected data is usually made available to supervisors and decision-makers, it has thus far not been made available to the CHWs themselves. As a result, CHWs often receive little to no feedback regarding their own work unless facilitated by a supervisor.

Our research addresses this gap by providing CHWs with personalized, on-demand feedback about their own work that is derived from the data that they have collected. Specifically, we worked with Accredited Social Health Activists (ASHAs), the largest cohort of CHWs in India, to create and deploy the ASHA Self-Tracking Application (ASTA). ASTA provides ASHAs with personalized information about their work through two interfaces that run in parallel: a web-based and a voice-based interface. The web-based interface consists of three visualizations: (1) a comparison graph that shows how many clients an ASHA has visited that month compared to a subset of her peers, providing an indication of progress towards the monthly goal of visiting all clients; (2) a calendar graph that shows the number of visits the ASHA made each day of the current month; and (3) a historical graph that shows the total number of clients visited each month for the past five months. The parallel voice-based system provides the same information as the comparison graph over a phone line, telling an ASHA how many clients she has visited out of the total number she has registered, and her current rank relative to a subset of her peers.

A prior paper [13] analyzed the impact of the system on ASHA performance, finding that ASHAs with access to the system made an average of $21.5 \%$ more client visits than ASHAs with access to a control system. However, the prior paper did not describe in detail or analyze the participatory design process used to create the system. It also did not ex- 
amine system usage behavior or discuss the ways in which each of the web- and voice-based systems, and the individual visualizations, may have motivated, supported, or discouraged ASHAs. To fill these gaps, this paper completely avoids discussing how the system affected ASHA performance and makes an entirely new set of research contributions.

First, we describe the in situ participatory design approach used to create the visualizations and interfaces that constitute the ASTA system. We highlight the complexities of designing graph-based feedback that can be understood by low-literate ASHAs who do not have high levels of education or experience with technology. Our experiences yield valuable insights and lessons learned that will be useful for others interested in designing interactive systems for low-literate populations in resource-constrained settings. Second, we present usage data from a 12-month deployment with 71 ASHAs in Uttar Pradesh, India. We show that ASHAs used both the web- and voice-based systems and reveal the benefits of providing more than one interaction modality. We also show that ASHAs used each of the three web-based visualizations for a variety of different reasons. Finally, we show how the comparative feedback provided by the system introduced elements of competition among ASHAs, which further motivated some ASHAs while leaving others feeling discouraged. Taken together, our findings suggest that giving ASHAs personalized feedback regarding their own work could be an effective way to motivate and support ASHA work processes.

\section{BACKGROUND AND RELATED WORK}

Our research contributes to a growing body of literature in HCI4D and ICTD that seeks to understand how computing technologies can be used to aid underserved populations around the world $[7,8,30]$. Focusing specifically on CHW programs, early work by DeRenzi et al. [11] showed that decision-support systems running on mobile PDA devices could help CHWs adhere to established medical protocols, while Florez at al. studied how mobile devices could be used to provide CHWs with digital job aids [15]. Ramachandran et al. [28] aimed to use mobile messaging and videos to improve health worker efficacy, noting the political, social, and cultural challenges associated with technology interventions that promote behavior change [27]. More recently, $\mathrm{Ku}-$ mar et al. [21] have explored equipping CHWs in India with community-led videos that they can use to promote behavior change. Their work stresses the importance of paying attention to local contexts and societal norms [20]. Learning from this prior work, our research takes an in situ, participatory design approach to try and ensure that the systems we create are usable and appropriate for the context.

Another set of relevant projects focus on sending reminder messages to CHWs or patients $[6,10,22]$. One particularly relevant project examined the role of supervisors in supporting CHW work [10]. The paper showed that informing a CHW's supervisor if she did not complete her work was an effective way to maintain CHW performance. However, when the communication to the supervisor was removed, the amount of work done by CHWs decreased. Our work takes a different approach in that, rather than involving the CHWs supervisor, the system attempts to motivate CHWs by providing them with useful information regarding the amount of work accomplished and the amount remaining.

All of the prior work described so far has enabled the oneway transfer of information (e.g., messages, videos) from a server or database to a CHW. Another set of projects enables the collection and one-way transfer of data in the opposite direction: from CHWs to a central database $[4,5,16,18]$. The collected data is usually made available to supervisors and decision-makers, including partner, donor, and government organizations. However, to date, the data collected by CHWs has not been made available to the CHWs themselves. As a result, CHWs usually receive little to no feedback regarding their own work unless facilitated by a supervisor. They have no way to analyze their past work or plan for future work. Our work addresses this gap by providing CHWs with ondemand information about their own work. This information is delivered to CHWs through a combination of voice-based feedback and web-based visualizations derived from the data the CHW herself has collected and submitted. To the best of our knowledge, we are the first to provide CHWs with personalized, on-demand feedback about their own work.

Finally, since many of the CHWs in our research had low levels of literacy, our design work draws heavily on research that examines how to create technologies for low-literate populations, including research that focuses on understanding the challenges faced by novice and low-literate technology users $[23,25]$, designing interfaces that have minimal text or are entirely text-free [24], combining audio and text interaction for semi-literate users [14], and creating voice-based systems that provide information to low-literate populations [26, 31]. Our designs incorporate many of the design recommendations provided by this body of work. Moreover, rather than choosing a single interaction modality, we chose to provide both voice- and web-based systems that operate in parallel.

\section{Background and Research Context}

This section provides background information regarding the ASHAs involved in our work and the context in which our research took place. We conducted our work within an existing mobile health program, the Reducing Maternal and Newborn Deaths (ReMiND) program [3] in Uttar Pradesh, India. The ReMiND program is being implemented by Catholic Relief Services (CRS) [29] in conjunction with Vatsalya [32], with the goal of improving maternal health and reducing neonatal mortality in Uttar Pradesh, which currently suffers critical shortages of health centers and health workers [2], and experiences high rates of maternal and infant mortality [1].

The ASHAs who participated in our research were already enrolled in the ReMiND program and had been trained to provide members of their communities with basic health services, counseling, and referrals. In keeping with the goals of the ReMiND program, the ASHAs that we worked with were particularly focused on maternal and newborn health. All ASHAs in the ReMiND program had previously been given Nokia C-2 feature phones running a CommCare application [12] that was designed to guide their work and aid data collection. Feature phones were chosen because they were locally 
available, affordable, familiar to many ASHAs, physically rugged, and had a long battery life. The CommCare application was in Hindi and contained localized images and audio prompts that helped ASHAs to register and monitor pregnant women. Supervisory staff then used the CommCare data submitted by ASHAs to monitor ASHA activity and make supportive visits based on performance. However, the CommCare application did not provide the ASHAs themselves with any information or feedback about their work. The visualizations that we designed and deployed in our research therefore represent the first time that ASHAs received information back from the system regarding their own work.

\section{Prior Work}

This is the second paper to be published on this research. The first paper [13] focused exclusively on analyzing the impact that the feedback and visualizations had on ASHA performance, measured as the number of client visits made by ASHAs. Findings from the paper showed that ASHAs with access to the system made an average of $21.5 \%$ more client visits than ASHAs with access to a control system. It also showed a correlation between system usage and performance.

However, although the prior paper briefly describes the design of the system, it does not analyze the diverse infrastructural, informational, and human challenges associated with designing usable and appropriate feedback mechanisms for ASHAs. It also does not provide lessons learned through the in-situ, participatory design approach that will be useful for other HCI4D researchers. Moreover, unlike the prior paper, this paper provides a detailed breakdown and analysis of system usage over a 12-month deployment, highlighting the benefits and limitations of each of the voice- and web-based systems. It also provides a rich, qualitative analysis of the ways in which each of the feedback systems and individual visualizations supported and motivated ASHA work processes. This detailed analysis of system usage, combined with qualitative data that reveals ASHA opinions, feelings, and reactions to the feedback, yields important new insights that will be valuable for the HCI4D and ICTD communities.

\section{SYSTEM DESIGN}

\section{Design Challenges}

In addition to the diverse environmental, infrastructural, and human challenges that result from working with low-literate ASHAs in rural India, our feedback mechanisms were built on top of an existing mobile health project and we needed to work within the constraints imposed by this project, including the devices already provided and information being collected.

\section{Device Constraints}

The feature phones provided to ASHAs presented substantial design constraints. For example, the devices had relatively small screen sizes (two inches) and low resolution (240 x 320 pixels). This gave us a very small physical and virtual area in which to design visualizations that provided useful information to ASHAs. We were also constrained by the limited functionality of the feature phone platform. Had we been able to design for a smartphone platform, it would have been straightforward to create a standalone application for the visualizations. Instead, we needed to create a web-based application that ran inside the browser (opera mini) and displayed high-quality PNG versions of the visualizations. It was also challenging to find an easy way for ASHAs to access their personalized website. Eventually we added a shortcut key to the home screen that opened the application, which paralleled a shortcut key that the ASHAs used to open CommCare.

\section{Information Constraints}

One decision that we needed to make early on was to define how the system would compute performance. We initially explored using a measure of visit quality, but quickly discovered that we were constrained by a need to use the data and metadata already being collected by the existing CommCare application. In light of this constraint, and to remain consistent with other aspects of the ASHAs supervision model, the key metric of visiting every pregnant client once per month became the focus. In addition to simplicity and ease of computation, this metric encourages ASHAs to register more clients and make more visits, both of which are desirable outcomes.

\section{Infrastructural and Environmental Challenges}

Most ASHAs worked in areas with relatively poor connectivity, so our intervention needed to work over a $2 \mathrm{G}$ network connection. It was also important that the visualizations not incur large data charges that would exceed the amount already provided to ASHAs through the ReMiND program. The physical working environment also presented a challenge since ASHAs frequently work outside in bright sunlight, which made it difficult to read detailed graphics or text.

\section{Human Challenges}

Perhaps the most important and complex set of challenges that we needed to coordinate were the needs of the ASHAs themselves. Most of these challenges stemmed from the fact that the ASHAs had variable-but generally low-education and literacy levels. The ASHAs had also had diverse levels of comprehension of graphs, written Hindi text, and more abstract visual metaphors. The main challenge was therefore to design visualizations that were intuitive for ASHAs and easily understandable with minimal training.

\section{Design Methodology}

Our design work involved a diverse range of literate and non-literate ASHAs and took place in two roughly sequential phases. In the first phase, we worked with ASHAs at multiple project sites, repeating the same process with each ASHA. At each site, we would begin by introducing (on paper) the concept of graphs and visualizations to ensure the ASHA understood visual representation of quantitative information. After introducing bar graphs, line graphs, and pie charts, we showed the ASHAs initial mockups of our visualizations (see Figure 1). An important part of the first phase was to learn how to quickly train ASHAs to read graphs. One effective strategy that we eventually converged on was to start by using the child health card, a familiar reference point for most ASHAs that also includes a graph. We also provided a variety of simple analogies to familiar topics, such as food or farming, to introduce and discuss graph concepts. In the second phase of our design work, we worked closely with a set of 
NUMBER OF VISITS

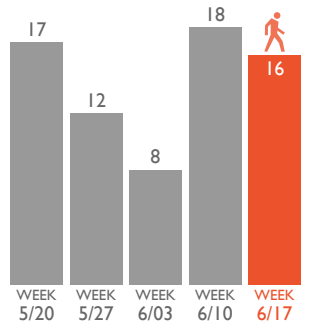

Figure 1. An early design used to elicit feedback during our iterative, participatory fieldwork.
My performance

1/7/2012- 29/7/2012

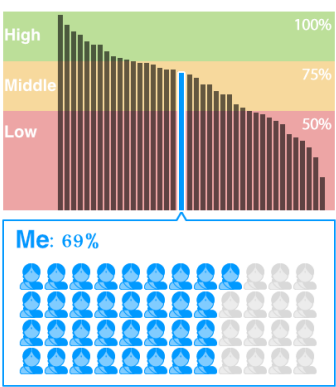

Figure 2. Intermediate design that incorporated colors from the child growth chart.
ASHAs in a neighboring block, following a rapid participatory design process to gather feedback on over 20 design iterations. During this iterative process we combined input from ASHAs, observations from field staff, and intuitions from the design team to create many new sets of visualizations before converging on the final set of visualizations that we deployed.

The voice-based system was created after the graphical system was complete. The design process for the voice system was short because we had already learned what information was understandable and appropriate for ASHAs. As a result, we were able to simply provide the information that was conveyed by the graphical system in spoken Hindi format. Before deployment, we pilot tested the voice system to ensure it was working properly and could be understood by ASHAs.

\section{Findings and Lessons from Design Iterations}

The initial designs we presented to the ASHAs in the first phase of our design process were simple and minimalist in an attempt to make them as understandable as possible. During the period of rapid iteration, we found that our designs increased in complexity as we tried to integrate different metaphors from ASHAs' day-to-day work. Our final set of visualizations then returned to a minimalist context-appropriate approach to communicating performance information.

\section{Interpretation is context-dependent}

We experienced numerous challenges creating visualizations that made sense to ASHAs. For example, our first set of visualizations used the color red to highlight the current day, but this led to confusion because, as we quickly discovered, red is used to highlight national holidays and weekends on Indian calendars. In an effort to match ASHAs existing work contexts, we also tried using the green, yellow, and red color coding from the child growth chart that they already use (see Figure 2). However, in some cases this backfired because ASHAs thought that the "you" label on the visualization was referring to a client and the colors showed the client's status. Another ASHA had a predetermined association with colors that came from a local rhyme. For example, to her green meant a beauty queen and yellow a "dirty fellow." Early iterations of the visualizations were developed with very rough approximations of workload, which resulted in challenges eliciting feedback from ASHAs because they became hyperfocused on the unrealistic workload. Finally, one iteration showed the percentage of clients visited, but one ASHA associated this with grades received in school, suggesting that the $71 \%$ in the example was just $4 \%$ less than a 'distinction'. All these findings highlight the importance of paying attention to the context and involving ASHAs in the design process.

\section{Training is a critical part of the process}

Training plays an enormous role in the success of technology interventions in low-resource contexts. In our case, it was necessary to train ASHAs not only on the technical components of accessing the visualizations, but also on the conceptual understanding of graphs. Our participatory design work made it clear that the concept of 'average' group performance was too abstract to be easily understood by the majority of the ASHAs without substantial training. It was much more clear to directly reference numbers. Similarly, there was confusion around labeling the ASHA's performance as 'me'. Instead, we discovered that it was better to use the terms 'you' and 'other ASHAs'. There are inherent tensions between making graphs immediately understandable and making graphs that contain rich information but that require training. Striking the correct balance so that all ASHAs can interpret a visualization with roughly one day of training takes iteration on both the graphs and the training methods.

\section{Different stakeholders have different priorities}

During our iterative design phase, ASHAs repeatedly asked us to create visualizations that included ASHA names so that they would know who was performing better than they were. This request revealed fundamental tensions between what the ASHAs wanted and what the implementing partner wanted (i.e., not shaming lower performing ASHAs). Balancing each stakeholder's priorities required compromise from all parties.

\section{It is beneficial to build on familiar concepts}

We found it beneficial to build on concepts that ASHAs were already familiar with. For example, during our fieldwork we noted that it was common for ASHAs to have calendars in their homes. Subsequently, the ASHAs responded favorably to our calendar visualizations, saying that they liked to be able to look back and see which days they had worked. In general, we tried to build on existing ASHA knowledge so that they would be comfortable with the resulting visualizations.

\section{The final visualizations}

The final set of visualizations are shown in Figure 3 (note that although the visualizations are shown in English for readability, the versions that we deployed with ASHAs were in Hindi). The comparison graph shows an ASHA's performance for that calendar month compared to a random subset of her peers. It consists of a basic histogram with qualitative labels on the $y$-axis. The visualization also contains repeated information. For example, the number of client visits an ASHA has made is conveyed by the histogram, the written text, and the pregnant women icons. The subset to which the ASHA was compared remained constant for the calendar month, but changed from month to month, which ensured that 


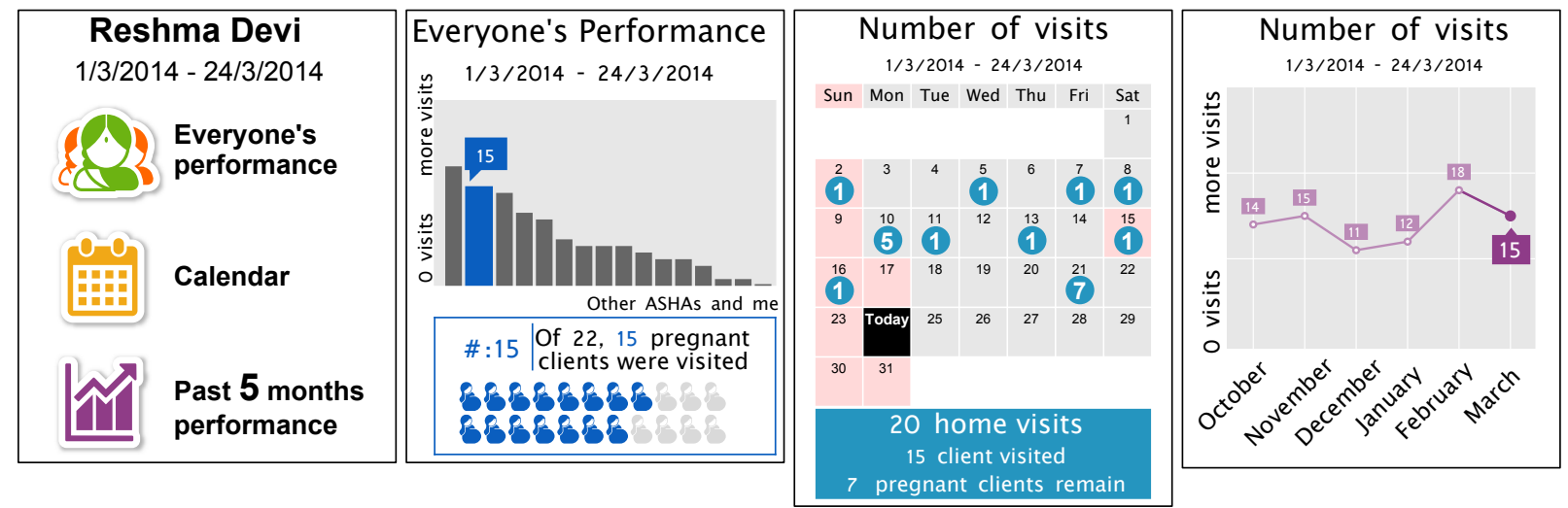

Figure 3. Screenshots from the final ASTA web system. Although we show the text in English here for readability the deployed application was in Hindi.

ASHAs did not get permanently stuck in either a high- or lowperforming group. The calendar graph shows the number of client visits the ASHA made each day of the month. Finally, the historical graph gives a sense of continuity, showing the number of visits made each month for the past five months.

\section{FIELD EVALUATION}

To evaluate the impact of the system on ASHAs, we conducted a 12-month deployment with 71 ASHAs in Uttar Pradesh, India. This section describes the ASHAs who participated in our study and our research methodology. The study was IRB approved by Maulana Azad Medical College, Harvard University, and the University of Washington.

\section{Participants}

We invited all the ASHAs in the ReMiND project to take part in our study and recruited a total of 71 ASHAs who participated for a period of 12 months (note that the ASHAs involved in the design phase of our research were from a different block and, to avoid the potential for bias, they did not take part in the study). All participants were female and ranged in age from 21 to 55 years $(M=32)$. In addition to their ASHA work, most ASHAs were also involved with agriculture $(69 \% ; n=49)$ or a non-agricultural family business $(11 \% ; n=8)$. Household sizes ranged from two to 19 , with a median size of six. The ASHAs came from a range of socioeconomic backgrounds, with self-reported individual monthly income ranging from 300 to 3000 INR (approx. US\$5 to $\$ 50)$. Participants reported relatively low levels of education, with 39\% having completed grade 10 and $7 \%$ having no formal schooling. Many of the ASHAs were also low-literate, with almost $31 \%(n=22)$ unable to easily read a basic Hindi sentence. Almost all participants $(96 \% ; n=68)$ had access to a mobile phone at home other than their work phone.

\section{Procedure}

We began by explaining the purpose of the study and answering any questions that ASHAs had. Then we interviewed participants to collect information regarding demographic characteristics, socioeconomic status, health knowledge, experience with technology, and prior work experience. All interviews were done in Hindi with data collected electronically before being translated into English and analyzed using Stata.
After completing the initial interview, ASHAs participated in a two-hour, in-person training session in which they were taught to use the system. The training session was designed to gradually introduce ASHAs to graph concepts. At the beginning of the session, the ASHAs were shown the growth charts that they used to monitor a child's growth as an example of how a graph can represent important information. Then, simple bar charts were introduced that compared the costs of different food items or the amount of milk sold by different individuals. As the concepts became clear, specific examples that were relevant to ASHA work were introduced. After practicing interpreting these graphs in a large paper format, the ASHAs were provided with access to our system on their phones and taught to interpret the visualizations. Finally, the ASHAs were trained to use the voice-based system.

After completing the training session, participants were free to interact with both the web- and voice-based systems as often as they chose. Participating ASHAs also received a weekly SMS reminder that encouraged them to check their information. Throughout the study a research assistant was available to assist ASHAs if they encountered technical issues or to resolve confusion. At the study midpoint (6 months), we interviewed all participants to understand their opinions and experiences using the system, check their devices were working, and provide supplementary training if necessary. At the end of the 12-month deployment we performed semistructured qualitative interviews with 19 participants. We interviewed a diverse subset of participants, including ASHAs with varying literacy levels and system usage patterns to create a representative sample of all participants. ASHAs were asked about their opinions of and reactions to the system, their preferences between the voice- and web-based systems, any confusion that they felt when looking at the visualizations, how the system affected their work processes and motivations, and their general usage habits. We also observed the ASHAs using the system and asked them questions about their understanding of the visualizations. In all of the interviews, we attempted to reduce participant response bias [9] by telling participants that negative feedback would be particularly useful and help us to improve the system, and by reminding them that their responses would remain anonymous. 


\section{Data Collection and Analysis}

We used a mixed methods approach in our study. Our quantitative data consists of system usage logs that recorded when and how often ASHAs accessed the system, which interaction modalities they used, how often they looked at each of the different visualizations, and so on. For the web-based system, we recorded a timestamp and the specific visualization(s) accessed. For the voice-based system, we recorded a timestamp, the length of the phone call, and how much of the recording the ASHA listened to before disconnecting. We also created the notion of an active "session", which we defined as a period of interaction with one of the systems that terminated after 10 minutes of inactivity. If an ASHA called into the voice-system multiple times within a 10-minute window, this was treated as one voice session. If, during that time, she also used the web system, it would be counted as one web session and one voice session. Our qualitative data consists of semi-structured interview data collected at the beginning, middle, and end of the study. All interviews were conducted in Hindi and translated into English for analysis. After translation, two of the authors independently performed open coding on the interview data to identify common patterns and recurring topics of interest. The authors then met and discussed the topics and codes that had emerged before further clustering the data into major themes. Following this, we conducted iterative analyses to further refine the themes and organize our prominent findings that we present below.

\section{FINDINGS}

The goal of our study was to analyze ASHA opinions, preferences, and system usage behaviors to understand the ways in which giving them access to feedback impacted their motivations and work processes. We structure our findings around four main questions that show (1) how the system supported ASHA work processes; (2) how ASHAs made use of both the web- and voice-based systems; (3) how ASHAs used each of the three web-based visualizations for different reasons; and (4) how ASHAs felt about the feedback they received.

\section{How did the system support ASHA work processes?}

The system usage $\log$ s show that ASHAs used the system throughout the study to obtain information about their work. Figure 4 depicts the total number of web- and voice-based sessions for each ASHA during the 12-month study. The horizontal lines on the graph show users who accessed the system at least weekly ( $n=40$ or $56 \%$ ) or five times per week ( $n=5$ or $7 \%$ ). Only three ASHAs accessed the system less than an average of once a month. Qualitative interview data suggests that the system supported ASHAs by providing them with useful information about their work and that the feedback motivated them to do more work. However, ASHAs were sometimes prevented from doing work by external factors beyond their control. In these cases, the feedback reminded them of their poor performance and made them feel bad. We now discuss these findings in detail.

\section{ASHAs found it useful to have information about their work} Our analysis revealed that the system supported ASHA work processes by providing them with useful information and feedback regarding their work, and many ASHAs described how they used the information provided by the system to understand their own productivity:

"[The system] is very good. It gives information about our work. It motivates us to do work. It is like a helper. And it also tells about the weakness in my work.” (P2)

The 'weakness' mentioned by this ASHA refers to the fact that, in showing ASHAs how many clients they had visited out of the total number that they had registered, the system implicitly highlights not only how much work they have done, but also how much work is still remaining for the month:

"The system tells us information about our work, like on which dates I made visits, my last 5 months performance, how many people I have met, how many are remaining - it gives me all the information." (P19)

Several ASHAs also mentioned that looking at the visualizations helped them to plan their time, and the system acted as a reminder that they needed to complete their ASHA duties:

"I feel good... I can know how much work I did. Like on 17 November I delivered a newborn so I couldn't make visits. But seeing the graph I think it's time to give a visit." (P3)

Being able to use the information provided by the system to plan their work was seen as a big improvement by many ASHAs, who said that previously they would just visit clients "according to our own motivation."

The system motivated ASHAs to do more work Many of the ASHAs we interviewed described how the information that they received from the system motivated them to do more work. In some cases, ASHAs were motivated to reach a good position within their comparison group:

"I liked seeing how much work I had completed and how far ahead I am. Ifelt motivated to move ahead to the next position and do more work." (P18)

In other cases, ASHAs explained that they were instead motivated to try and achieve the greatest number of client visits by registering as many pregnant women as possible:

"I felt motivated ... like I completed eight home visits so by seeing this it motivated me to do more work. It also motivated me to add new pregnant women in my lists because then I can do more home visits." (P4)

This finding validates our choice to use the total number of client visits as the primary metric rather than the percentage of clients visited, which could incentivize ASHAs to register fewer women to achieve a higher percentage. Overall, the fact that the system motivated ASHAs to register more pregnant women is encouraging and highlights the potential for the system to positively impact ASHA work processes.

\section{External challenges prevented ASHAs from doing their work} Although many ASHAs said that the system motivated them to do more work, several told us they felt discouraged when they were prevented from doing work by external factors out of their control. For example, many ASHAs and clients were 


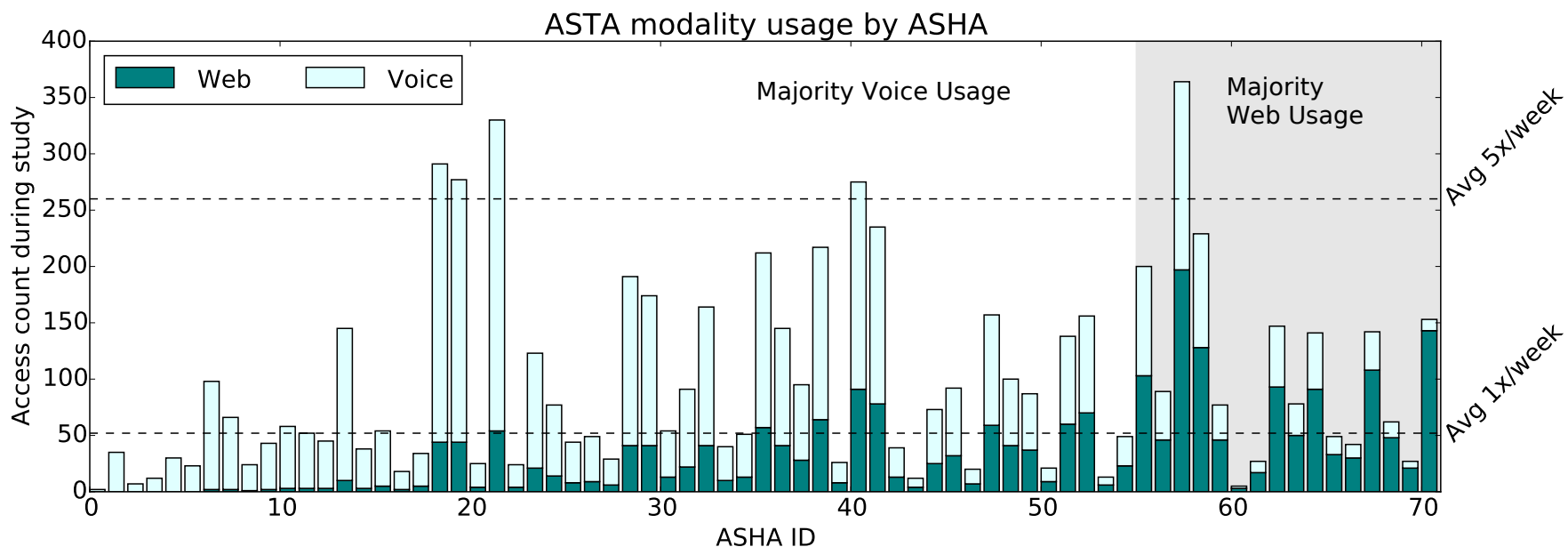

Figure 4. Individual ASHA usage of the voice and web systems during the study. The ASHAs are ordered from greater portion of voice-based use to greater portion of web-based use. The shaded region represents majority web use and the white region represents majority voice use. The two horizontal lines represent an average of weekly and daily (weekdays) system usage.

heavily involved in farming and these responsibilities sometimes prevented them from participating in ASHA visits:

"I feel bad when I cannot do much work. When I go to the home visits many people are not available because of harvesting which is why my work is less." (P5)

Several ASHAs also described how they often did not receive the money that was owed to them by the government and as a result they were not motivated to work. In these cases, the system continued to show the ASHA that she was performing poorly, which often made the ASHA feel bad despite her performance being justified by lack of compensation.

How did ASHAs use the web- and voice-based systems? Figure 4 depicts the total number of usage sessions for each ASHA over the 12-month study. At a high level, the system usage data reveals that most ASHAs made use of both the web- and voice-based systems. The ASHAs in Figure 4 are ordered from greater portion of voice-based use to greater portion of web-based use. The shaded region on the right represents the tipping point where ASHAs used the webbased system for the majority of their sessions. Most ASHAs $(n=55,77.5 \%)$ used the voice system more frequently than the web system. We now discuss the reasons ASHAs gave for preferring one system over another and why it was beneficial to provide more than one way to interact with the system.

Benefits and limitations of the web-based system

The main reason ASHAs gave for preferring the web system over the voice system was because the three visualizations provided a richer, more detailed description of their work:

"I like [the web-based system] more because there are three programs, like calendar, comparison, and previous five months, while the [voice-based] system only tells my position and the number of home visits." (P12)

In addition to providing a greater volume of information, ASHAs also appreciated that they were able to look at the visualizations for as long as they liked, which gave them time to interpret the graphs at their own pace. By contrast, the voice-based system delivered information at a constant pace and, if ASHAs missed some of the information, they would need to repeat the entire message. Some of the ASHAs also perceived that the voice-based system was provided primarily for illiterate ASHAs and that if they knew how to read they should rather use the web-based system:

"I like [the web system] because I have no difficulty reading. I think the [voice system] is for people who can't read." (P1)

Despite this perception, we found that the proportion of lowliterate ASHAs in the majority web usage group and majority voice usage group was roughly equivalent $(31.25 \%$ vs. $30.9 \%$ respectively), which suggests that literacy level did not play a significant role in determining system preference.

The main limitation of the web-based system that the ASHAs described was that the small size of the screen made it difficult to read and interpret the graphs. Moreover, when we asked the ASHAs to show us how they used the web-based system, we found that several of them were unable to read some of the numbers on the screen, which they explained was due to their poor eyesight and the "very, very small" size of the numbers.

\section{Benefits and limitations of the voice-based system}

The main reasons that ASHAs gave for preferring the voicebased system over the web-based system was that IVR was "easier to use and takes less time." ASHAs simply needed to leave a missed call and the system called them back:

"I like the [voice system]. You push the two button and you hear all of the information. I can use it as often as I want and it doesn't use my balance. And it is easy to use." (P14)

In addition to being easier to use, several ASHAs said that they liked the voice-based system because listening to information was more familiar for them than reading graphs. Several mentioned that they already used their phones for listening to songs, and so it made more sense to them to also listen to the information provided by the system. The voice-based 
system was also particularly useful for ASHAs who were illiterate, had poor eyesight, or who otherwise had difficulty interpreting the web-based visualizations.

The main limitations of the voice-based system were that it provided a relatively small amount of information compared to the web-based system and that, if ASHAs needed anything repeated, they had to listen to the entire message again. One ASHA also complained that she would have liked the automated voice-based system to respond and engage in conversation with her, telling us, "She always just tells you things, but she never listens or talks with you." (P9)

\section{It was beneficial to provide multiple interaction modalities}

The fact that most ASHAs used both the web-based and the voice-based systems suggests that it was beneficial for them to have more than one way of accessing information about their work. Many ASHAs explained that although they generally preferred to use the web-based system, they would use the voice-based system "if I have less time or if the network is poor." Others told us that they would use the voice-based system if their phone's battery was low or if they experienced technical difficulties accessing the web-based visualizations.

Several ASHAs pointed out that when a phone has zero balance, it is not possible to make a missed call to access the voice system. Similarly, when the data bundle expires, it is not possible to access the web system, though this was rare in practice. In either case, ASHAs would use their second choice system until the research assistant was able to address their barrier to access. These findings speak to the benefits of having more than one way to access information. Some ASHAs also liked to compare the information provided by the two systems to see if it matched, "I like to cross check the visual and voice systems to see if they are consistent" (P5). Finally, several ASHAs said that they used both systems because they wanted to try all of the options, while others felt obliged to use both simply because both had been provided.

\section{How did ASHAs use the three web-based visualizations?}

In addition to analyzing how ASHAs used the web- and voice-based interfaces, we were also interested to understand how they used the three different web-based visualizations. Figure 5 shows the proportion that each ASHA accessed each of the three visualizations as a percentage of the total number of times they used the web-based system. All but one of the ASHAs made use of all three visualizations during the study period, which is encouraging and suggests that each of the visualizations served a different purpose and provided ASHAs with different information. Overall, the comparison graph was used the most, followed by the calendar graph, with the historical graph used the least. In summary, 34 ASHAs accessed the comparison graph more than the other two visualizations, 23 accessed the calendar view the most, and 10 accessed the historical view the most, with four ASHAs accessing each of the visualizations equally. We now discuss the perceived value of the three web-based visualizations and the reasons that ASHAs gave for using each visualization.

\section{Reasons for using the comparison graph}

The main reasons that ASHAs gave for liking the comparison graph was that it showed them their position in the group, the amount of work they had done, and the amount of work remaining. Several ASHAs liked the visual depiction of the quantity of work completed, with one ASHA describing how the bar increased as she worked "like a bucket filling with water until it is full and you are done." Another ASHA explained how the visual appearance of the bar chart helped to make the information understandable even for illiterate ASHAs:

"Those ASHAs who cannot read, when they see the graph they can still know how much work they have completed and how much remains." (P10)

\section{Reasons for using the calendar graph}

Many of the ASHAs who liked the calendar view did so because they used calendars in their daily lives and were already familiar with the calendar-based information. The calendar view also clearly showed which days were holidays, and ASHAs expressed that they liked knowing when they were allowed to skip work. However, some ASHAs told us that they found the calendar view challenging to interpret and so they would ignore the calendar visualization:

"I used the calendar the least because I cannot easily understand it. So I do not spend much time on it. I just open it and then go on to the next page." (P15)

This finding highlights the benefits of providing a variety of different visualizations, since any one graph is unlikely to fit the needs and constraints of all ASHAs.

\section{Reasons for using the historical graph}

The historical graph showed the number of visits an ASHA made each month for the last five months, and many ASHAs liked that this graph provided a sense of prior work activities:

"I like the last five month performance because it is like a stair that my work is going up and down. I can see how many more [visits] I did and how many fewer." (P16)

ASHAs also described how this graph encouraged them to plan for the future, with several telling us how they would use information about their performance during the past month to plan their work for the next month. However, the ASHAs also told us that although they appreciated the historical information, they did not need to look at it as often as the other graphs since the information changed relatively infrequently:

"I don't look at the [historical graph] often because it doesn't change. You can look at it once and you don't need to look again. I look at the calendar and my performance more to see my information." (P10)

\section{How did ASHAs feel about the feedback they received?}

We chose to provide ASHAs with comparative feedback that shows their number of visits relative to a subset of their peers. This comparison introduced competitive elements among the ASHAs and different participants reacted to these elements in different ways. In addition to introducing competition, the feedback also prompted discussion among ASHAs and generally increased their awareness of each others' work. 


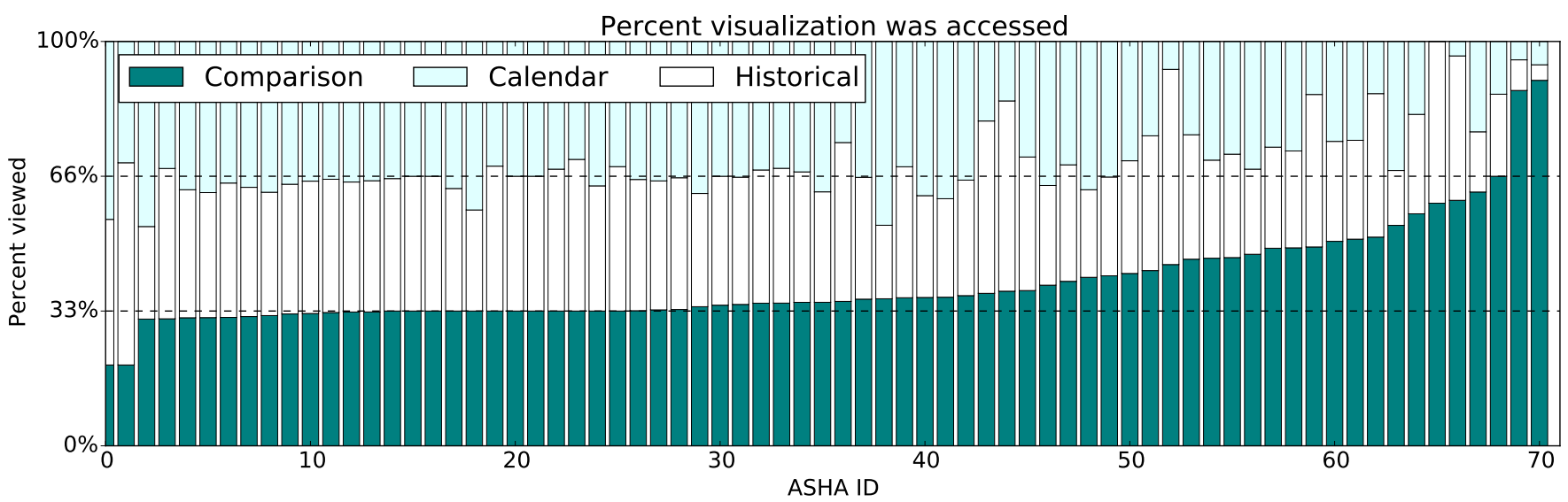

Figure 5. Individual ASHA usage of the three different web-based visualizations. The ASHAs are ordered by increasing use of the comparison graph.

\section{Comparative feedback introduced competitive elements}

Much of the data obtained from interviews with ASHAs suggested that the comparative feedback provided by the system introduced competitive elements to ASHA work. However, the effects that these competitive elements had on ASHAs differed between individuals. For example, some ASHAs told us that they were not affected by the competitive elements and that they simply focused on trying to do their work, claiming, "I am only competing with myself". Other ASHAs were highly competitive and went so far as to blame the system or other external factors for even a small drop in their performance. For example, one competitive ASHA told us:

"I was always in the first place, except one time I was second, maybe because there was a problem with the network." (PI)

Many ASHAs explained that the competitive elements introduced by the system had a positive effect on their work by motivating them to visit more clients:

"I liked the [comparison graph] better because I could tell what my position was. I treated it as a competition which motivated me to do more work.” (P4)

Unsurprisingly, ASHAs who achieved a high level of performance, and therefore a high overall position, said that the feedback provided by the system made them feel good about themselves. Many ASHAs also equated their position with the amount of work they had done, describing how if they were in first position, that meant that they had done 'enough' work, but if they were in last position it meant they should be working harder. However, the competitive elements introduced by our comparative feedback certainly did not work for all of the ASHAs, and in some cases had the adverse effect of making ASHAs feel bad about their work:

"It feels bad to see you are in the last position. After completing my work I was still in last position. I do not have very many pregnant women in my area." (P15)

As this ASHA points out, our choice to use the total number of visits made by an ASHA as the primary performance metric disadvantages ASHAs who work in areas with few pregnant clients. We chose this metric so that ASHAs would not be incentivized to register fewer clients but if an ASHA happens to not have many pregnant clients in her area, she will not be able to make many visits, and her overall performance will be low through no fault of her own. The fact that the feedback ended up discouraging some ASHAs is a negative consequence that we plan to address through future research (discussed in detail in our Discussion section).

The system prompted discussion among ASHAs

Many ASHAs described how the feedback provided by the system made them more aware of the other ASHAs operating in their area and prompted group discussions. Many ASHAs requested that we provide them with the names of the ASHAs in their comparison group so that they would know who they were being compared against. However, there was concern that publicly revealing the names of ASHAs who were performing poorly may further discourage them. Several ASHAs also described how they would get together as a group and discuss the information provided by the system. In addition to comparing their performance to each other by showing each other their personalized graphs, some ASHAs would use the group discussions to try and motivate lowperforming ASHAs to do their work. For example, one of the high-performing ASHAs explained:

"Sometimes we discuss and I ask other ASHAs - who are these ASHAs in the bottom position? I explain to them - do your work so you are not in the bottom position." (P2)

Another example of ASHAs trying to help each other occurred when one ASHA used her device to play feedback for another ASHA who was experiencing technical difficulties:

"One time I played the IVR for another ASHA because hers was not working but mine was.” (P12)

Unfortunately, since the voice-based feedback played by the system was personalized to the ASHA calling in, the information would only be relevant to that ASHA and would not have helped the ASHA experiencing technical difficulties. Nevertheless, it is encouraging that the system motivated ASHAs to help and support other ASHAs in their area. 


\section{DISCUSSION}

Our analysis of how ASHAs used the system, combined with qualitative data that reveals ASHA opinions, feelings, and reactions to the feedback, demonstrates the value of providing ASHAs in low-resource environments with personalized, ondemand information that is derived from the data they have collected. Our findings and analysis also highlight rich opportunities for future research that focuses on designing and deploying new feedback mechanisms that may better support and motivate field workers in low-resource contexts. We now discuss some of the key takeaways from our work.

Designing feedback mechanisms and visualizations for lowliterate ASHAs who do not have high levels of education or experience with technology required a large amount of patience, iteration, and in situ participatory design. Our experiences echo those described in a recent review of the HCI4D literature by Dell and Kumar [8], which points out, "Just because the physical infrastructure has improved doesn't mean the human infrastructure has improved." In our case, the technological infrastructure in India has improved to make it feasible to deploy two-way mobile communication systems in which ASHAs collect and submit data to the system, and the system in turn provides feedback to ASHAs. However, even though the technological infrastructure exists, a large amount of iterative design work was required to create a system that enables the humans in this ecosystem - the ASHAs - to understand and interpret the information provided. One key takeaway from our work is therefore that it demonstrates the importance of HCI4D researchers interested in designing for low-literate populations to spend time in the field ensuring that their system is delivering information that is relevant, understandable, and appropriate for the context.

Our work also highlights how new technologies should not be thought of in isolation, but rather in the context of a wide range of external factors that might impact ASHA work processes. For example, our findings show that ASHAs sometimes failed to receive the monetary compensation owed to them by the government and so were disincentivized from doing work. At other times, ASHAs and/or their clients needed to spend their time harvesting crops and so were unable to participate in home visits. All these factors make up the broad ecosystem in which the technology is being used and it is important to consider this larger context when designing, deploying, and evaluating new technology interventions.

Another key takeaway from our work centers on the nature of the comparative feedback provided by our system. Many of the ASHAs who participated in our study found it useful to receive feedback regarding their work and used the information provided to reflect on their own work practices, evaluate themselves, coordinate their activities, and plan for the future. However, in choosing to provide ASHAs with feedback that compared them to a subset of their peers, we also introduced competitive elements among the ASHAs, and our findings show that this competition affected ASHAs in different ways, motivating some participants while leaving others feeling discouraged. To overcome this limitation, we plan to conduct future research that explores a range of alterna- tive feedback mechanisms that may appeal to ASHAs who are motivated in different ways. For example, we could explore more nuanced ways of measuring ASHA performance, such as by assigning different numbers of 'points' for different tasks. Using such a system, an ASHA who maybe has fewer clients registered, but whose clients engage in desired health behaviors, could still achieve high performance.

In addition, comparative feedback is certainly not the only kind of feedback that could be provided. For example, we plan to explore mechanisms that may encourage ASHAs by promoting teamwork [17], which may appeal to ASHAs who prefer collaboration over competition. Teams that achieve certain goals, or that are most improved, could then be publicly acknowledged by, for example, awarding a 'team of the month'. Moreover, we plan to explore how we might tailor the feedback provided to an individual ASHA based on personality types that could be identified through baseline surveys. For example, prior work suggests that personality traits may be tied to different motivational criteria for performance [19]. By identifying in advance whether or not an ASHA will be better motivated by, for example, competition or collaboration, we will be able to ensure that individual ASHAs receive feedback that is supportive and encouraging.

Finally, our work clearly highlights the benefits of providing participants with more than one way to interact with the system. The majority of ASHAs made use of both the web- and voice-based systems, with different ASHAs preferring each of the systems for different reasons. In addition, having multiple systems provided redundancy that enabled ASHAs to still access information even if one of the systems happened to be unavailable. We therefore recommend that other researchers working in low-resource contexts consider providing participants with multiple ways of interacting with the system.

\section{CONCLUSION}

This paper describes the design and deployment of mobile voice- and web-based systems that aim to support ASHAs by providing them with feedback regarding their work. We described the in-situ, participatory design approach that we used to create feedback systems that were appropriate and usable for low-literate ASHAs. We then described usage data from a 12-month deployment of the system with 71 ASHAs in India. We showed that the system supported ASHA work processes by providing motivation and information that helped their work. We also performed a detailed analysis of system usage and described ASHA opinions and feelings regarding the feedback that they received. Taken together, our findings yield valuable insights and lessons learned that will be useful to anyone interested in designing and deploying technologies with underserved populations in low-resource contexts.

\section{ACKNOWLEDGMENTS}

We thank all our ASHA participants for their time, feedback, and the important work they do; Vatsalya and CRS for being strong collaborators; and the research assistants for their hard work. This research was funded by the Massachusetts General Hospital Consortium for Affordable Medical Technologies (CAMTech) and NSF Grant No. IIS-1111433. 


\section{REFERENCES}

1. 2011. Census of India. http: //censusindia.gov.in/2011-prov-results/ paper2/data_files/UP/7-pop-12-22.pdf. (2011).

2. 2012. Uttar Pradesh State Profile. http://nrhm.gov.in/nrhm-in-state/ state-wise-information/uttar-pradesh.html. (2012).

3. 2013. Baseline Study Summary: ReMiND. Technical Report. Catholic Relief Services.

4. Matt Berg, James Wariero, and Vijay Modi. 2009. Every child counts: The use of SMS in Kenya to support the community based management of acute malnutrition and malaria in children under five. Columbia University.

5. Evan Borkum, Anitha Sivasankaran, Swetha Sridharan, Dana Rotz, Sukhmani Sethi, Mercy Manoranjini, Lakshmi Ramakrishnan, and Anu Rangarajan. 2015. Evaluation of the Information and Communication Technology (ICT) Continuum of Care Services (CCS) Intervention in Bihar. Technical Report. Mathematica Policy Research.

6. C Bourne, V Knight, R Guy, H Wand, H Lu, and A McNulty. 2011. Short message service reminder intervention doubles sexually transmitted infection/HIV retesting rates among men who have sex with men. Sexually transmitted infections 87, 3 (2011), 229-231.

7. Marshini Chetty and Rebecca E. Grinter. 2007. HCI4D: $\mathrm{HCI}$ Challenges in the Global South. In $\mathrm{CHI}$ '07 Extended Abstracts on Human Factors in Computing Systems (CHI EA '07). 2327-2332.

8. Nicola Dell and Neha Kumar. 2016. The Ins and Outs of HCI for Development. In Proceedings of the 2016 CHI Conference on Human Factors in Computing Systems (CHI' '16). 2220-2232.

9. Nicola Dell, Vidya Vaidyanathan, Indrani Medhi, Edward Cutrell, and William Thies. 2012. "Yours is Better!": Participant Response Bias in HCI. In Proceedings of the SIGCHI Conference on Human Factors in Computing Systems (CHI '12). ACM, New York, NY, USA, 1321-1330.

10. Brian DeRenzi, Leah Findlater, Jonathan Payne, Benjamin Birnbaum, Joachim Mangilima, Tapan Parikh, Gaetano Borriello, and Neal Lesh. 2012. Improving Community Health Worker Performance Through Automated SMS. In International Conference on Information and Communication Technologies and Development (ICTD '12). 25-34.

11. Brian DeRenzi, Neal Lesh, Tapan Parikh, Clayton Sims, Werner Maokla, Mwajuma Chemba, Yuna Hamisi, Marc Mitchell, and Gaetano Borriello. 2008. e-IMCI: Improving pediatric health care in low-income countries. In SIGCHI conference on human factors in computing systems. ACM, 753-762.
12. Brian DeRenzi, Clayton Sims, Jonatham Jackson, Gaetano Borriello, and Neal Lesh. 2011. A framework for case-based community health information systems. In Global Humanitarian Technology Conference (GHTC), 2011 IEEE. IEEE, 377-382.

13. Brian DeRenzi, Jeremy Wacksman, Nicola Dell, Neal Lesh, Gaetano Borriello, and Scott Lee. 2016. Closing the Feedback Loop: A 12-month Evaluation of ASTA, a Self-Tracking Application for ASHAs. In International Conference on Information and Communication Technologies and Development (ICTD '16).

14. Leah Findlater, Ravin Balakrishnan, and Kentaro Toyama. 2009. Comparing Semiliterate and Illiterate Users' Ability to Transition from Audio+Text to Text-only Interaction. In SIGCHI Conference on Human Factors in Computing Systems (CHI '09). 1751-1760.

15. Jose F Florez-Arango, M Sriram Iyengar, Kim Dunn, and Jiajie Zhang. 2011. Performance factors of mobile rich media job aids for community health workers. Journal of the American Medical Informatics Association 18, 2 (2011), 131-137.

16. Carl Hartung, Yaw Anokwa, Waylon Brunette, Adam Lerer, Clint Tseng, and Gaetano Borriello. 2010. Open Data Kit: Building Information Services for Developing Regions. In International Conference on Information and Communication Technologies and Development (ICTD). 10.

17. Guido Hertel, Udo Konradt, and Borris Orlikowski. 2004. Managing distance by interdependence: Goal setting, task interdependence, and team-based rewards in virtual teams. European Journal of work and organizational psychology 13, 1 (2004), 1-28.

18. M Sriram Iyengar, Jose F Florez-Arango, and Carlos A Garcia. 2009. GuideView: a system for developing structured, multimodal, multi-platform persuasive applications. In International Conference on Persuasive Technology. ACM, 31.

19. Timothy A Judge and Remus Ilies. 2002. Relationship of personality to performance motivation: a meta-analytic review. Journal of applied psychology 87, 4 (2002), 797.

20. Neha Kumar and Richard J. Anderson. 2015. Mobile Phones for Maternal Health in Rural India. In Proceedings of the 33rd Annual ACM Conference on Human Factors in Computing Systems (CHI '15). ACM, New York, NY, USA, 427-436.

21. Neha Kumar, Trevor Perrier, Michelle Desmond, Kiersten Israel-Ballard, Vikrant Kumar, Sudip Mahapatra, Anil Mishra, Shreya Agarwal, Rikin Gandhi, Pallavi Lal, and Richard Anderson. 2015. Projecting Health: Community-led Video Education for Maternal Health. In Proceedings of the Seventh International Conference on Information and Communication Technologies and Development (ICTD '15). ACM, New York, NY, USA, Article 17, 10 pages. 
22. Walley J. Katabira E. Muchuro S. Balidawa H. Namagala E. Ikoona E. Kunutsor, S. 2010. Using mobile phones to improve clinic attendance amongst an antiretroviral treatment cohort in rural Uganda: a crosssectional and prospective study. AIDS and behavior 14, 6 (2010), 1347-1352.

23. Indrani Medhi, Somani Patnaik, Emma Brunskill, S.N. Nagasena Gautama, William Thies, and Kentaro Toyama. 2011. Designing Mobile Interfaces for Novice and Low-literacy Users. ACM Trans. Comput.-Hum. Interact. 18, 1 (May 2011).

24. Indrani Medhi, Aman Sagar, and Kentaro Toyama. 2006. Text-free user interfaces for illiterate and semi-literate users. In Information and Communication Technologies and Development, 2006. ICTD'06. International Conference on. IEEE, 72-82.

25. Maletsabisa Molapo and Gary Marsden. 2013. Software Support for Creating Digital Health Training Materials in the Field. In International Conference on Information and Communication Technologies and Development: Full Papers - Volume 1 (ICTD '13). 205-214.

26. Neil Patel, Deepti Chittamuru, Anupam Jain, Paresh Dave, and Tapan S. Parikh. 2010. Avaaj Otalo: A Field Study of an Interactive Voice Forum for Small Farmers in Rural India. In SIGCHI Conference on Human Factors in Computing Systems (CHI'10). 733-742.

27. Divya Ramachandran, John Canny, Prabhu Dutta Das, and Edward Cutrell. 2010a. Mobile-izing Health Workers in Rural India. In SIGCHI Conference on Human Factors in Computing Systems (CHI'10). 1889-1898.

28. Divya Ramachandran, Vivek Goswami, and John Canny. 2010b. Research and Reality: Using Mobile Messages to Promote Maternal Health in Rural India. In Proceedings of the 4th ACM/IEEE International Conference on Information and Communication Technologies and Development (ICTD '10). ACM, New York, NY, USA, 35:1-35:10.

29. Catholic Relief Services. 2016. www.crs.org. (2016).

30. Kentaro Toyama. 2010. Human-Computer Interaction and Global Development. Foundations and Trends in Human-Computer Interaction 4, 1 (2010), 1-79.

31. Aditya Vashistha, Edward Cutrell, Gaetano Borriello, and William Thies. 2015. Sangeet Swara: A Community-Moderated Voice Forum in Rural India. In SIGCHI Conference on Human Factors in Computing Systems (CHI'15).

32. Vatsalya. 2016. www.vatsalya.org. (2016). 